\title{
Association between autoimmune pancreatitis and malignancy
}

\author{
Kenji Hirano $\cdot$ Hiroyuki Isayama $\cdot$ Minoru Tada $\cdot$ \\ Kazuhiko Koike
}

Received: 20 March 2014/ Accepted: 25 March 2014/Published online: 18 April 2014

(C) Springer Japan 2014

\begin{abstract}
Chronic inflammatory diseases are often associated with malignancy, and this phenomenon occurs even in the field of gastroenterology. For example, chronic pancreatitis has been reported to be highly associated with the occurrence of pancreatic cancer. However, the association between autoimmune pancreatitis (AIP) and pancreatic cancer is still an unsolved problem. As AIP is associated with immunoglobulin G4-related disease, which causes a variety of inflammatory and fibrotic lesions, the association between AIP and total malignancies is also a matter of interest. Here, we discuss the association between AIP and malignancy. As for the association between AIP and pancreatic cancer, there is no conclusive evidence, although several reports have suggested that the relative risk of pancreatic cancer is high in AIP. Concerning the association between AIP and total malignancies, there are two reports from Japan. Neither report supported the idea that chronic inflammation in AIP caused the increased risk of total malignancies. However, one report proposed the possibility that AIP might develop as a paraneoplastic syndrome in some patients because many cancers occurred within 1 year of AIP diagnosis, and thereby concluded that patients with AIP were at high risk of having various cancers. The paraneoplastic syndrome hypothesis should be tested in future studies.
\end{abstract}

Keywords Autoimmune pancreatitis - Malignancy . Pancreatic cancer $\cdot K$-ras mutation · Paraneoplastic syndrome $\cdot \operatorname{IgG} 4$-related disease

K. Hirano $(\bowtie) \cdot$ H. Isayama $\cdot$ M. Tada $\cdot$ K. Koike Department of Gastroenterology, Graduate School of Medicine, The University of Tokyo, 7-3-1 Hongo, Bunkyo-ku,

Tokyo 113-8655, Japan

e-mail: khirano-tky@umin.ac.jp

\author{
Abbreviations \\ AIP Autoimmune pancreatitis \\ Ig Immunoglobulin \\ IgG4-RD IgG4 related disease \\ RA Rheumatoid arthritis \\ SIR Standardized incidence rate \\ CI Confidence interval \\ PanIN Pancreatic intraepithelial neoplasia \\ EUS-FNA Endoscopic ultrasound-guided fine needle \\ aspiration \\ IPMN Intraductal papillary mucinous neoplasm
}

\section{Introduction}

Chronic inflammatory diseases are often associated with the occurrence of malignancy, and this occurs even in the field of gastroenterology. For example, esophageal adenocarcinoma, gastric cancer, hepatocellular carcinoma, biliary carcinoma, and colorectal cancer are associated with reflux esophagitis and Barrett's esophagus, Helicobacter pylori gastritis, viral hepatitis and nonalcoholic steatohepatitis, primary sclerosing cholangitis, and ulcerative colitis, respectively. The relative risk of pancreatic cancer in patients with chronic pancreatitis, excluding autoimmune pancreatitis (AIP), is reported to be high (2.3-26.7) [1-3]. Thus, AIP could be associated with a high risk of pancreatic cancer. Moreover, AIP is regarded as a pancreatic lesion of immunoglobulin G4-related disease (IgG4-RD), causing inflammatory and fibrotic lesions in various organs $[4,5]$. Therefore, it may be a matter of interest whether AIP is related to an increased risk of malignancy.

Rheumatoid arthritis (RA) is associated with disorders in various organs, such as the lung and skin. A recent study 
of 3,771 patients with RA in the UK found the overall standardized incidence ratio (SIR) for total malignancy to be 1.28 [95 \% confidence interval (CI) 1.10-1.48], which was significantly high [6]. On the basis of this finding, the same theory might be true of AIP or IgG4-RD. Although the relationship between AIP and malignancy remains unsolved, we discuss several viewpoints below.

\section{AIP and pancreatobiliary cancer}

Suspected association between AIP and pancreatobiliary cancer

Although several reports have suggested that the relative risk of pancreatic cancer is high in AIP, there is no conclusive evidence. According to Kamisawa et al., high-frequency $K$-ras mutations $(2+$ or $3+$ ) were detected in the pancreatic ductal epithelium of all eight resected patients with AIP in their study. Moreover, high-frequency $K$-ras mutations were detected in the epithelium of the common bile duct $(56 \%, 5 / 9)$ and the gallbladder (44\%, 4/9) [7]. The infiltration of Foxp3 + regulatory $\mathrm{T}$ cells (Treg) is observed not only in AIP but also in pancreatic cancer [8]. It is also reported that Treg cells are required for K-rasmediated lung tumorigenesis in mice [9]. These findings suggest the possibility of a close association between AIP and pancreatobiliary cancer. Gupta et al. [10] inspected resected pancreata to clarify the prevalence of pancreatic intraepithelial neoplasia (PanIN) in 28 cases of AIP, and identified that 23 cases ( $82 \%$ ) showed PanIN; grades 2 and 3 PanIN were demonstrated in 7 (25\%) cases and 1 case, respectively, thus strongly indicating an elevated risk of pancreatic malignancy.

On the other hand, Khalid et al. [11] analyzed pancreatic specimens obtained using endoscopic ultrasound-guided fine needle aspiration (EUS-FNA) from 11 cases of pancreatic cancer and 14 cases of AIP. They reported that the $K$-ras mutation occurred in none of the AIP cases and $10 / 11$ cases with pancreatic cancer. Although this seems to contradict the findings of Kamisawa et al., methodological differences in obtaining pancreatic specimens may explain the differences (i.e., resection or EUS-FNA). For example, because of the dilution effect, the amount of specimens obtained by EUS-FNA may be too small to detect the K-ras mutation in pancreatic ductal epithelium. However, it should be noted that high-frequency $K$-ras mutations do not equate to the high frequencies of pancreatic cancer.

The rate of pancreatobiliary cancer in AIP

Many authors have reported the long-term prognosis of AIP, together with the prevalence of associated malignancies. According to an analysis of 563 AIP cases from 17 Japanese institutes, pancreatic cancer occurred at a rate of $0.53 \%(3 / 563)$ [12]. We investigated the abstracts submitted to Japan Digestive Disease Week 2010 (JDDW 2010) and determined that the prevalence of pancreatic cancer and biliary cancer was $1.1 \%$ (5/446 in 9 institutes), and $1.2 \%$ (4/345 in 7 institutes), respectively. There was a large difference among institutes. At one institute, pancreatic cancer was observed in $5 \%$ (3/60) of cases, whereas there were no reports at six institutes. As the exact follow-up period was unknown, the evaluation of this data is difficult. However, these prevalences do not seem markedly high.

It will be adequate to evaluate the occurrence rate using the SIR. If a standard group in which age, sex, and observation period are matched with an AIP patient group, then we can calculate the expected number of malignancies from the supposed standard group. The SIR is the 'observed number of malignancies from an AIP group divided by the expected number of malignancies from a supposed standard group'. In short, the SIR reflects how many times patients with AIP suffer from malignancies compared to a standard population. If the analysis targets the total malignancy rate among patients with AIP, the sample size of the study need not be large because of the higher number of total malignancies. However, if the analysis targets only pancreatic cancer among patients with AIP, a considerably larger sample size will be necessary owing to the smaller incidence. Even if the SIR of pancreatic cancer from AIP is significantly high, it would not be as high as that from chronic pancreatitis or intraductal papillary mucinous neoplasm (IPMN), which has a SIR of 10.7 [13]. As for IPMN, pancreatic cancer was discovered in $2.6 \%$ (17/642) during an average follow-up of 4.8 years in one study [13]. If 100 patients with AIP are necessary to identify one case of pancreatic cancer and the SIR is 3.6, as per our previous report [14], a sample of approximately 450 patients is necessary to demonstrate statistical significance [15]. It is possible that the SIR is much $<3.6$ [16]. Thus, large-scale nationwide research is required to resolve this problem.

\section{AIP, IgG4-RD, and total malignancies}

Suspected associations between AIP and total malignancy

Both AIP and RA are systemic inflammatory diseases. If RA is associated with the increased risk of total malignancy, the same may apply to AIP. In addition to chronic inflammation, diabetes mellitus is often associated with AIP due to pancreatitis and steroid treatment. In these 
patients, the relative risks of pancreatic cancer, hepatocellular carcinoma, colorectal cancer, breast cancer, and ureteral cancer are increased [17]; this may suggest an increased risk of malignancy in AIP. Kamisawa et al. [18] reported that high-frequency $K$-ras mutations were detected in the gastric mucosa $(2 / 3)$ and colonic mucosa $(2 / 3)$, and suggested that patients with AIP might have risk factors for gastric and colon cancer.

\section{Reported prevalence of total malignancy in AIP or IgG4-RD}

In the literature, three papers (all from Japan) have evaluated the rate of total malignancy in AIP and IgG4-RD using the SIR. Yamamoto et al. [19] first suggested an increased risk of total malignancy in IgG4-RD. They followed up 106 patients with IgG4-RD, including 69 with Mikulicz's disease and 10 with AIP, for an average of 3.1 years. They reported malignancy in 11 patients $(10.4 \%)$ with a SIR for total malignancy of 3.83 , which was significantly high.

Shiokawa et al. [16] followed up 108 patients with AIP for a median of 3.3 years. They identified 18 malignancies in 15 patients (13.9\%), and reported a SIR of 2.7 (95\% CI 2.4-3.9), which was significantly high, comprising gastric cancer $(n=7)$, lung cancer $(n=3)$, malignant lymphoma $(n=2)$, prostate cancer $(n=2)$, colon cancer $(n=2)$, bile duct cancer $(n=1)$, and thyroid cancer $(n=1)$. Interestingly, there was no pancreatic cancer in their series. In addition, AIP and malignancy were comorbid in eight cases. Thus, the SIRs of cancer in the first and subsequent years after AIP diagnosis were 6.1 (95\% CI 2.3-9.9) and 1.5 (95\% CI 0.3-2.8), respectively, indicating that the occurrence of cancer was significantly higher in the first year. This result contradicts the notion that long-term inflammation causes malignancy. With regard to this point, they suggested that immunological reaction to cancer might cause AIP, i.e., AIP might develop as a paraneoplastic syndrome in some patients.

On the other hand, we previously concluded that IgG4$\mathrm{RD}$ was not associated with an increased incidence of total malignancy [14]. In our experience of 126 patients with IgG4-RD, there were 23 malignancies among 21 patients, which might not be a small figure. However, malignancy and IgG4-RD occurred concurrently (within 6 months) in seven patients. We excluded these seven patients together with another six patients whose follow-up period was $<6$ months. As a result, we analyzed 113 patients, of which 95 had AIP. They were followed up for an average of 6.1 years, with 15 malignancies occurring in 14 patients [lung cancer $(n=5)$, pancreatic cancer $(n=2)$, gastric cancer $(n=2)$, bile duct cancer $(n=1)$, renal cancer
Table 1 Comparison of papers analyzing the association between IgG4-RD and total malignancy

\begin{tabular}{|c|c|c|}
\hline Author & $\begin{array}{l}\text { Shiokawa et al. } \\
{[16]}\end{array}$ & $\begin{array}{l}\text { Hirano et al. } \\
{[14]}\end{array}$ \\
\hline Number of cases & 108 & $113^{\mathrm{a}}$ \\
\hline Number of AIP cases & $108(100 \%)$ & $95(84 \%)$ \\
\hline Occurrence of malignancy & $\begin{array}{l}18 \\
\text { malignancies } \\
\text { in } 15 \text { patients }\end{array}$ & $\begin{array}{l}14 \\
\text { malignancies } \\
\text { in } 15 \\
\text { patients }^{\mathrm{b}}\end{array}$ \\
\hline $\begin{array}{l}\text { How to deal with patients in } \\
\text { whom IgG4-RD and } \\
\text { malignancies occurred } \\
\text { concurrently }\end{array}$ & $\begin{array}{l}\text { Included ( } 8 \\
\text { cases) }\end{array}$ & $\begin{array}{l}\text { Excluded }(7 \\
\text { cases) }\end{array}$ \\
\hline Follow-up period & $\begin{array}{l}3.3 \text { years } \\
(\text { median })\end{array}$ & $\begin{array}{r}6.1 \text { years } \\
(\text { mean })\end{array}$ \\
\hline $\begin{array}{l}\text { SIR for total malignancies (95\% } \\
\text { CI) }\end{array}$ & $2.7(1.4-3.9)$ & $\begin{array}{l}1.04 \\
\quad(0.57-1.75)\end{array}$ \\
\hline $\begin{array}{l}\text { SIR for total malignancies one or } \\
\text { more years after the occurrence } \\
\text { of IgG4-RD }\end{array}$ & $1.5(0.3-2.8)$ & $\begin{array}{l}0.90 \\
\quad(0.47-1.56)\end{array}$ \\
\hline $\begin{array}{l}\text { SIR for total malignancies when } \\
\text { patients in whom IgG4-RD and } \\
\text { malignancies occurred } \\
\text { concurrently are included }\end{array}$ & - & $\begin{array}{l}1.56 \\
(0.99-2.34)\end{array}$ \\
\hline $\begin{array}{l}\text { Suggested cause for the presence } \\
\text { of many patients in whom } \\
\text { IgG4-RD and malignancies } \\
\text { occurred concurrently }\end{array}$ & $\begin{array}{l}\text { Paraneoplastic } \\
\text { syndrome }\end{array}$ & $\begin{array}{l}\text { Institutional } \\
\text { bias }\end{array}$ \\
\hline
\end{tabular}

IgG4-RD IgG4 related disease, AIP autoimmune pancreatitis, SIR standardized incidence ratio

a 7 cases in which IgG4-RD and malignancy occurred concurrently and 6 cases whose follow-up period was $<6$ months were excluded b In a total of 126 IgG4-RD patients, 23 malignancies occurred in 21 patients

$(n=1)$, breast cancer $(n=1)$, tongue cancer $(n=1)$, malignant melanoma $(n=1)$, and acute myeloid leukemia $(n=1)$ ]. The SIR for total malignancy was $1.04(95 \% \mathrm{CI}$ $0.57-1.75)$.

Our conclusions apparently contradict those of Shiokawa et al. However, the difference may result from differences in the methods of analysis (Table 1). Because Shiokawa et al. considered the possibility of AIP as a paraneoplastic syndrome, they included concomitant AIP and malignancy. Because our interest was only to investigate if IgG4-RD caused malignancy, we excluded these patients. We consider that the evaluation of the association between IgG4-RD and malignancy occurring concurrently is difficult. It is because institutional bias may exist concerning patients with concurrent IgG4-RD and malignancy; for example, most patients present with malignant diseases at cancer hospitals. Close examination may reveal that asymptomatic IgG4-RD is diagnosed by chance in some patients with malignancy because both IgG4-RD and 
cancer are frequently observed in the elderly. In other words, if an institute has more cancer patients, the likelihood of IgG4-RD and malignancy being diagnosed concurrently increases. At present, we believe it is difficult to judge whether the association between IgG4-RD and malignancy is accidental or due to a paraneoplastic syndrome. Therefore, we excluded such patients from our analysis and focused on if IgG4-RD caused malignancy.

Despite the different conclusions between our work and that of Shiokawa et al., there is some agreement. Shiokawa et al. also mentioned that the SIR for malignancy $\geq 1$ year after the occurrence of AIP was 1.5 (95\% CI 0.3-2.8), which was not significant. Our conclusion may support this. In short, both studies do not support the hypothesis that IgG4-RD causes malignancy. However, concerning patients with concurrent IgG4-RD and malignancy, Shiokawa et al. consider the possibility of paraneoplastic syndrome (i.e., that malignancy causes IgG4-RD). Once again, we emphasize the possibility of institutional bias; from our data, we cannot confirm or deny the authenticity of the paraneoplastic syndrome hypothesis.

This report is limited by the fact that specifying the onset time of IgG4-RD or AIP is sometimes difficult. If there were prodromal symptoms before the final diagnosis of IgG4-RD, the timing of the appearance of the symptoms was regarded as the onset time in our study [14]. However, in patients without any symptoms, the onset time was regarded as the time of diagnosis. For cases of malignancy, the time of diagnosis was used for the onset time. It is difficult to determine the exact time of onset of either IgG4-RD or malignancy. In addition, depending on which is investigated, the association between $\operatorname{IgG} 4-\mathrm{RD}$ and malignancy or between AIP and malignancy, data analysis may differ. For example, in a patient diagnosed with Mikulicz's disease who develops concurrent AIP and malignancy after 5 years, it follows that malignancy occurred during follow-up of the IgG4-RD, but at the same time as AIP. It is difficult to know how to deal with such a patient during statistical analysis.

\section{Conclusions}

Although an association between AIP and pancreatic cancer has been suggested, it has not been evaluated using the SIR. If the association is significant, the SIR for pancreatic cancer in AIP may be lower than in chronic pancreatitis or IPMN. Thus, a large investigation is necessary to clarify this. Concerning AIP and total malignancy, it is also difficult to draw any specific conclusions given our current understanding. In light of the results of two reports [14, 16], however, it seems unlikely that AIP is responsible for the increased risk of total malignancy. Shiokawa et al. suggested that AIP might develop as a paraneoplastic syndrome in some patients. This hypothesis should be tested in future studies.

\section{Disclosures}

Conflict of Interest: Kenji Hirano, Hiroyuki Isayama, Minoru Tada, and Kazuhiko Koike declare that they have no conflict of interest.

Human/Animal Rights: All procedures followed were in accordance with the ethical standards of the responsible committee on human experimentation (institutional and national) and with the Helsinki Declaration of 1975, as revised in 2008(5).

Informed Consent: Informed consent was obtained from all patients for being included in the study.

\section{References}

1. Bansal P, Sonnenberg A. Pancreatitis is a risk factor for pancreatic cancer. Gastroenterology. 1995;109:247-51.

2. Talamini G, Falconi M, Bassi C, Sartori N, Salvia R, Caldiron E, et al. Incidence of cancer in the course of chronic pancreatitis. Am J Gastroenterol. 1999;94:1253-60.

3. Malka D, Hammel P, Maire F, Rufat P, Madeira I, Pessione F, et al. Risk of pancreatic adenocarcinoma in chronic pancreatitis. Gut. 2002;51:849-52.

4. Shimosegawa T, Chari ST, Frulloni L, Kamisawa T, Kawa S, Mino-Kenudson $\mathrm{M}$, et al. International consensus diagnostic criteria for autoimmune pancreatitis: guidelines of the International Association of Pancreatology. Pancreas. 2011;40:352-8.

5. Umehara H, Okazaki K, Masaki Y, Kawano M, Yamamoto M, Saeki T, et al. Comprehensive diagnostic criteria for IgG4-related disease (IgG4-RD), 2011. Mod Rheumatol. 2012;22:21-30.

6. Mercer LK, Davies R, Galloway JB, Low A, Lunt M, Dixon WG, et al. Risk of cancer in patients receiving non-biologic diseasemodifying therapy for rheumatoid arthritis compared with the UK general population. Rheumatology (Oxford). 2013;52:91-8.

7. Kamisawa T, Tsuruta K, Okamoto A, Horiguchi S, Hayashi Y, Yun $\mathrm{X}$, et al. Frequent and significant $\mathrm{K}$-ras mutation in the pancreas, the bile duct, and the gallbladder in autoimmune pancreatitis. Pancreas. 2009;38:890-5.

8. Moo-Young TA, Larson JW, Belt BA, Tan MC, Hawkins WG, Eberlein TJ, et al. Tumor-derived TGF-[beta]mediates conversion of CD4 + Foxp 3 + regulatory $\mathrm{T}$ cells in a murine model of pancreas cancer. J Immunother. 2009;32:12-21.

9. Granville CA, Memmott RM, Balogh A, Mariotti J, Kawabata S, Han W, et al. A central role for Fozp3 + regulatory T cells in K-ras-driven lung tumorigenesis. PLoS ONE. 2009;4:e5061.

10. Gupta R, Khosroshahi A, Shinagare S, Fernandez C, Ferrone C, Lauwers GY, et al. Does autoimmune pancreatitis increase the risk of pancreatic carcinoma? A retrospective analysis of pancreatic resections. Pancreas. 2013;42:506-10.

11. Khalid A, Dewitt J, Ohori NP, Chen JH, Fasanella KE, Sanders $\mathrm{M}$, et al. EUS-FNA mutational analysis in differentiating autoimmune pancreatitis and pancreatic cancer. Pancreatology. 2011;11:482-6.

12. Kamisawa T, Shimosegawa T, Okazaki K, Nishino T, Watanabe $\mathrm{H}$, Kanno A, et al. Standard steroid treatment for autoimmune pancreatitis. Gut. 2009;58:1504-7.

13. Kawakubo K, Tada M, Isayama H, Sasahira N, Nakai Y, Yamamoto $\mathrm{K}$, et al. Incidence of extrapancreatic malignancies in 
patients with intraductal papillary mucinous neoplasms of the pancreas. Gut. 2011;60:1249-53.

14. Hirano K, Tada M, Sasahira N, Isayama H, Mizuno S, Takagi K, et al. Incidence of malignancies in patients with $\mathrm{IgG} 4$-related disease. Intern Med. 2014;53:171-6.

15. Ury HK, Wiggins AD. Another shortcut method for calculating the confidence interval of a Poisson variable (or of a standardized mortality ratio). Am J Epidemiol. 1985;122:197-8.

16. Shiokawa M, Kodama Y, Yoshimura K, Kawanami C, Mimura J, Yamashita Y, et al. Risk of cancer in patients with autoimmune pancreatitis. Am J Gastroenterol. 2013;108:610-7.

17. Shikata K, Ninomiya T, Kiyohara Y. Diabetes mellitus and cancer risk: review of the epidemiological evidence. Cancer Sci. 2013;104:9-14.
18. Kamisawa T, Horiguchi S, Hayashi Y, Yun X, Yamaguchi T, Tsuruta K, et al. K-ras mutation in the major duodenal papilla and gastric and colonic mucosa in patients with autoimmune pancreatitis. J Gastroenterol. 2010;45:771-8.

19. Yamamoto M, Takahashi H, Tabeya T, Suzuki C, Naishiro Y, Ishigami $\mathrm{K}$, et al. Risk of malignancies in IgG4-related disease. Mod Rheumatol. 2012;22:414-8. 